\title{
Análisis estructural de chasis prototipo para automóvil tipo SAE BAJA
}

\section{Structural analysis of prototype chassis for SAE BAJA vehicle}

\author{
HUERTA-GAMEZ, Hector $\dagger^{*}$, HERRERA-OLIVARES, Edgar Daniel, ZUÑIGA-CERROBLANCO, \\ José Luis y AGUILAR-MORENO, Antonio Alberto
}

Universidad Politécnica de Juventino Rosas, México.

ID $1^{\text {er }}$ Autor: Hector, Huerta-Gamez / ORC ID: 0000-0002-5088-310X, CVU CONACYT ID: 373690

ID $1^{\mathrm{er}}$ Coautor: Edgar Daniel, Herrera-Olivares / ORC ID: 0000-0002-5776-0313

ID $2^{\text {do }}$ Coautor: José Luis, Zuñiga-Cerroblanco / ORC ID: 0000-0003-0493-8197, CVU CONACYT ID: 208410

ID $3^{\text {er }}$ Coautor: Antonio Alberto, Aguilar-Moreno / ORC ID: 0000-0002-7652-5925, CVU CONACYT ID: 254188

DOI: $10.35429 /$ JOIE.2020.13.4.12.19

\begin{abstract}
Resumen
En el presente trabajo se desarrolla el análisis numérico de la estructura de un prototipo de chasis para automóvil tipo SAE BAJA. Dentro de la propuesta del modelo se consideran factores que se vuelven importante al momento de implementarlo, dichos factores son: seguridad al conductor, espacios suficientes para los componentes del vehículo, entre otros puntos básicos. Así mismo, el modelo es sometido a diferentes pruebas establecidas por la Sociedad de Ingenieros Automotrices (SAE, siglas en inglés), pruebas como: Impacto frontal, impacto trasero, impacto lateral, volcadura, pruebas de aterrizaje por salto delantero y por salto trasero; las condiciones a las cuales son sometidas estas pruebas son extraídas de artículos (pruebas) y aplicadas en las zonas adecuadas para tener los resultados más precisos y cercanos a la realidad. El análisis se realiza con diferentes materiales y perfiles utilizados para miembros estructurales, de esta forma también se obtiene la configuración adecuada de estos parámetros. Debido a los parámetros satisfactorios obtenidos, se tiene como resultado un modelo bastante eficiente, esto repercute en la seguridad necesaria para el conductor, básicamente, debido a que el sistema cuenta bajas deformaciones en los materiales y soportando las tensiones relativamente altas.
\end{abstract}

Recibido Enero 18, 2020; Aceptado Marzo 31, 2020

\begin{abstract}
In the present work, the numerical analysis of the structure of a prototype of a SAE BAJA type automobile chassis is developed. Within the proposal of the model, factors that become important when implementing it are considered, these factors are: driver safety, sufficient spaces for vehicle components, among other basic points. Likewise, the model is subjected to different tests established by the Society of Automotive Engineers (SAE), tests such as: Front impact, rear impact, side impact, overturn, landing tests for front jump and rear jump; the conditions to which these tests are subjected are extracted from articles (tests) and applied in the appropriate areas to have the most accurate and close to reality results. The analysis is carried out with different materials and profiles used for structural members, in this way the appropriate configuration of the profiles and materials is also obtained. Due to the satisfactory parameters obtained, a fairly efficient model is obtained, this has an impact on the necessary safety for the driver, basically, because the system has low deformations in the materials and withstands relatively high stresses.
\end{abstract}

Citación: HUERTA-GAMEZ, Hector, HERRERA-OLIVARES, Edgar Daniel, ZUÑIGA-CERROBLANCO, José Luis y AGUILAR-MORENO, Antonio Alberto. Análisis estructural de chasis prototipo para automóvil tipo SAE BAJA. Revista de Ingeniería Innovativa. 2020. 4-11:12-19.

\footnotetext{
*Correspondencia al Autor (Correo Electrónico: hhuerta_ptc@upjr.edu.mx)

$\dagger$ Investigador contribuyendo como primer autor.
} 


\section{Introducción}

BAJA SAE es un evento para estudiantes universitarios de ingeniería, organizado por la Sociedad de Ingenieros Automotrices. El evento organizado en nombre de la competencia MiniBAJA sirve como una plataforma para que los jóvenes estudiantes de ingeniería muestren sus habilidades al diseñar, fabricar y validar un vehículo todo terreno de una sola plaza y adquirir una experiencia de la vida real mientras superan obstáculos y desafíos.

El chasis del vehículo es la estructura de soporte principal de un vehículo BAJA SAE, en él se montan todos los demás componentes del vehículo. La función principal de un chasis es soportar todos los componentes mecánicos del vehículo y soportar cargas estáticas y dinámicas, sin deformarse. Debe diseñarse de manera ergonómica y eficiente, con un costo y peso óptimos, además debe de ser un diseño muy seguro para el conductor.

\section{Material Utilizado}

La selección del material adecuado es un punto importante a considerar, ya que de él dependerán los resultados de la simulación, en la tabla 1 , se muestran los diferentes materiales con los que se trabajó dentro de la simulación.

\begin{tabular}{|l|r|r|r|}
\hline & \multicolumn{1}{|c|}{ AISI 1020 } & \multicolumn{1}{c|}{ AISI 1018 } & \multicolumn{1}{c|}{ AISI 4130 } \\
\hline $\begin{array}{l}\text { Módulo } \\
\text { Elástico }\end{array}$ & $2 \times 10^{11} \mathrm{~Pa}$ & $2.05 \times 10^{11} \mathrm{~Pa}$ & $2.05 \times 10^{11} \mathrm{~Pa}$ \\
\hline $\begin{array}{l}\text { Coeficiente de } \\
\text { Poisson }\end{array}$ & 0.29 & 0.29 & 0.29 \\
\hline $\begin{array}{l}\text { Densidad de } \\
\text { Masa }\end{array}$ & $7900 \mathrm{~K} / \mathrm{m}^{3}$ & $7870 \mathrm{~K} / \mathrm{m}^{3}$ & $7850 \mathrm{~K} / \mathrm{m}^{3}$ \\
\hline $\begin{array}{l}\text { Límite } \\
\text { Elastico }\end{array}$ & $351 \mathrm{MPa}$ & $370 \mathrm{MPa}$ & $460 \mathrm{MPa}$ \\
\hline
\end{tabular}

Tabla 1 Propiedades Mecánicas Aceros AISI

Fuente: elaboración propia [Word]

Un factor importante dentro del desarrollo del proyecto es la selección del perfil adecuado, dentro del software de simulación, existen diversos perfiles, en el caso del proyecto, se trabajó con el perfil cuadrado, el cual se muestra en la figura 1 , dicho perfil tiene las dimensiones que se muestran en la tabla 2.

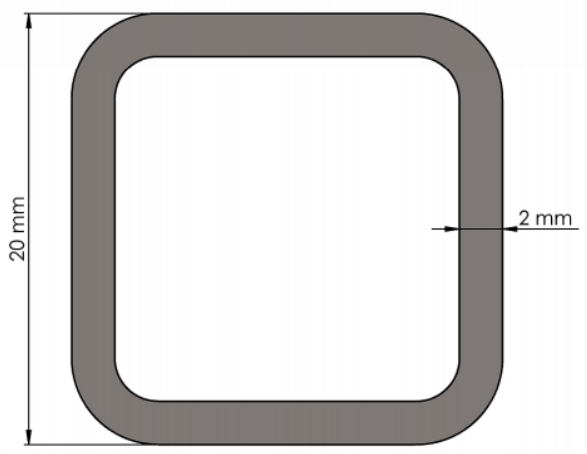

Figura 1 Perfil de trabajo

Fuente: Elaboración propia [Solidworks]

\begin{tabular}{|l|r|}
\hline \multicolumn{1}{|r|}{ Perfil Cuadrado } \\
\hline Lado & $20 \mathrm{~mm}$ \\
\hline Espesor & $2 \mathrm{~mm}$ \\
\hline
\end{tabular}

Tabla 2 Dimensiones del perfil de trabajo Fuente: elaboración propia [Word]

\section{Análisis}

Después de haber seleccionado el perfil para el chasis, fue necesario someter a pruebas de esfuerzo y resistencia toda la armadura bajo condiciones severas. El fin de poder soportar los impactos a los que se somete es que proporcionará mucha mayor seguridad para el conductor sin sufrir deformaciones significativas.

Las pruebas a las que sometió el chasis fueron las siguientes:

\section{Impacto Frontal \\ 2. Impacto Trasero \\ 3. Impacto Lateral \\ 4. Impacto Por Volcadura}

\section{Impacto Por Salto Delantero}

\section{Impacto Por Salto Trasero}

El modelo del chasis se realizó considerando que éste fuera lo más seguro posible, de ahí que se utilizaron geometrias triangulares, dicho modelo se muestra en la figura 2: 


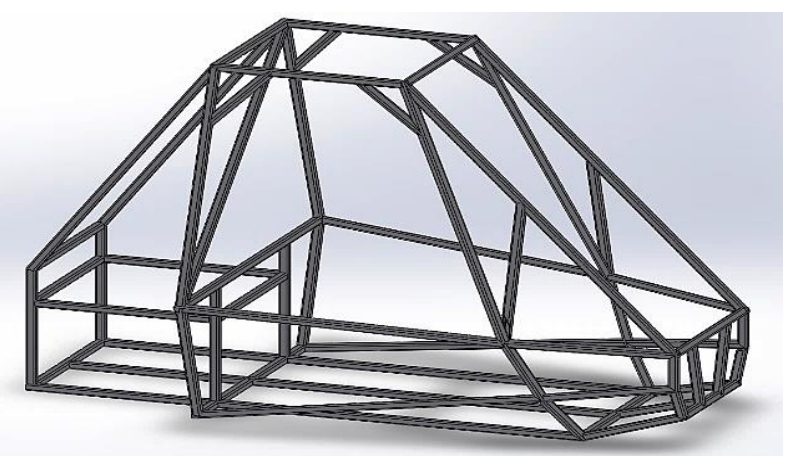

Figura 2 Modelo del chasis

Fuente: Elaboración propia [Solidworks]

\section{Impacto Frontal}

Para calcular la fuerza a aplicar en el impacto frontal se utilizó la masa total del chasis + masa total del motor + masa total de la batería. La suma de todas ellas se multiplicó por una fuerza $\mathrm{G}$ de 4 de acuerdo con la fomula de BAJA SAE. $\mathrm{F}=$ ma $140 * 4 * 9.1=5500 \mathrm{~N}$ (aprox) aplicamos $5500 \mathrm{~N}$ en la parte frontal del chasis divididas en 4 fuerzas de $1375 \mathrm{~N}$ c/u, se realizaron 6 sujeciones de geometría fija en la parte trasera del chasis. La figura 3, muestra las condiciones de carga a las cuales el modelo fue sometido para llevar a cabo la simulación de cargas por impacto frontal.

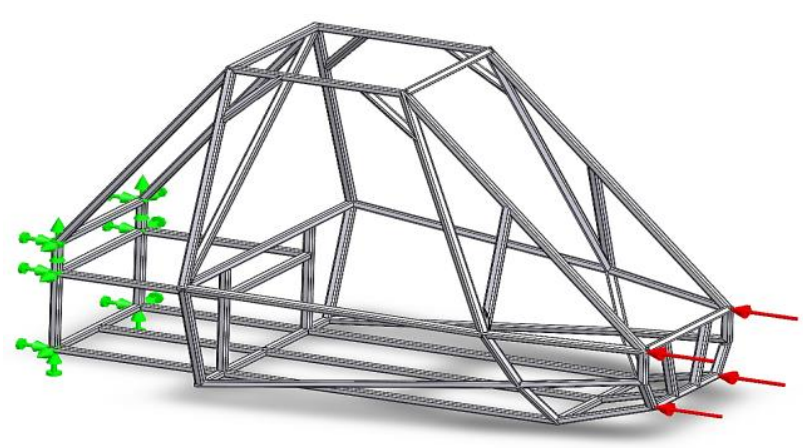

Figura 3 Condiciones de carga para simulacion de impacto frontal.

Fuente: elaboración propia [Solidworks]

En la figura 4, se muestra la zona donde los desplazamientos debido al impacto frontal repercutieron en mayor medida, así mismo, numéricamente se observaron los siguientes parámetros en el caso de la deformación máxima: $3.68 \mathrm{~mm}$ en el material AISI 1020, 3.59 $\mathrm{mm}$ en el material AISI 1018 y $3.59 \mathrm{~mm}$ en el material AISI 4130.
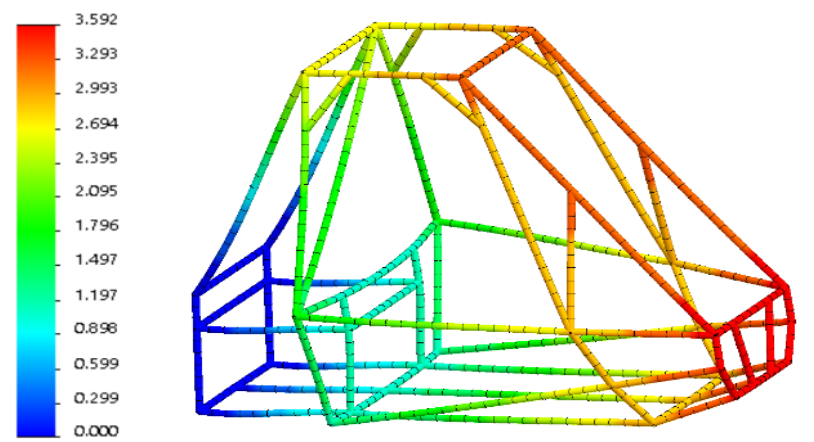

Figura 4 Desplazamientos debido al impacto frontal Fuente: elaboración propia [Solidworks]

También, se obtuvieron los esfuerzos debido al impacto frontal; la figura 5 muestra las zonas en las cuales el sistema está más esforzado, así cmo también se observaron los siguientes parámetros: un esfuerzo máximo de 102.4 MPa en la estructura quedando a un $29 \%$ en el material AISI 1020, $27 \%$ en el material AISI 1018 y $22 \%$ en el material AISI 4130 de su límite elástico.
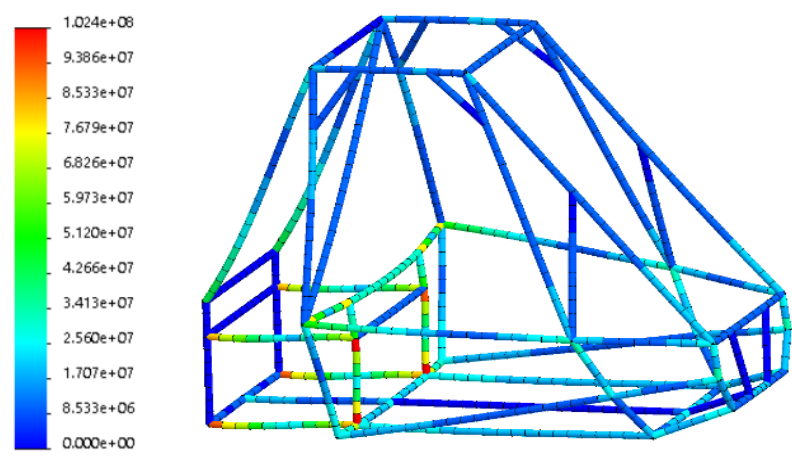

Figura 5 Esfuerzos debido al impacto frontal Fuente: elaboración propia [Solidworks]

\section{Impacto Trasero}

Para calcular la fuerza a aplicar en el impacto trasero se utilizó la masa total del chasis + masa total del motor + masa total de la batería. La suma de todas ellas se multiplicó por una fuerza $\mathrm{G}$ de 4 de acuerdo a la fomula de BAJA SAE. $\mathrm{F}=\mathrm{ma} 140 * 4 * 9.1=5500 \mathrm{~N}$ (aprox) aplicamos $5500 \mathrm{~N}$ en la parte trasera del chasis divididas en 4 fuerzas de $1375 \mathrm{~N} \mathrm{c/u}$, se realizaron 4 sujeciones de geometría fija en la parte delantera del chasis. En la figura 6 se muestran las condiciones de carga a las cuales fue sometido el chasis para simular el impacto trasero. 


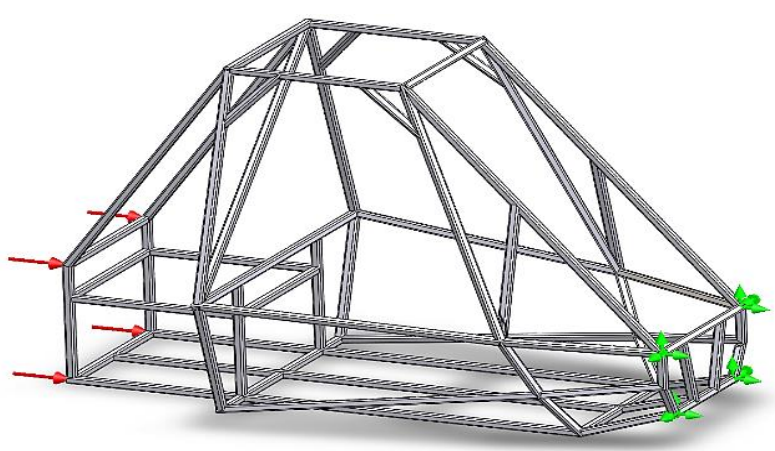

Figura 6 Condiciones de carga debido al impacto trasero Fuente: elaboración propia [Solidworks]

En la figura 7 se observa cómo son los desplazamientos del chasis bajo las condiciones de carga debido al impacto trasero; en dicho análisis se obtuvo que la máxima deformación fue de $1.61 \mathrm{~mm}$ en el material AISI 1020, $1.57 \mathrm{~mm}$ en el material AISI 1018 y $1.57 \mathrm{~mm}$ en el material AISI 4130.
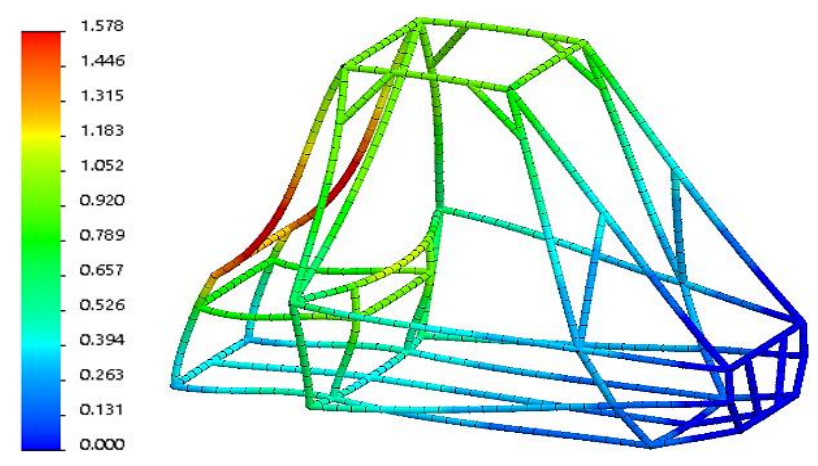

Figura 7 Desplazamientos debido al impacto trasero Fuente: elaboración propia [Solidworks]

En la figura 8 se observan las zonas en las cuales el chasis se encuentra más esforzado debido a las condiciones de carga consideradas para el impacto trasero; de la misma manera se obtuvo que el esfuerzo máximo fue de 107.1MPa en la estructura quedando a un $30 \%$ en el material AISI 1020, $28 \%$ en el material AISI 1018 y $23 \%$ en el material AISI 4130 de su límite elástico.
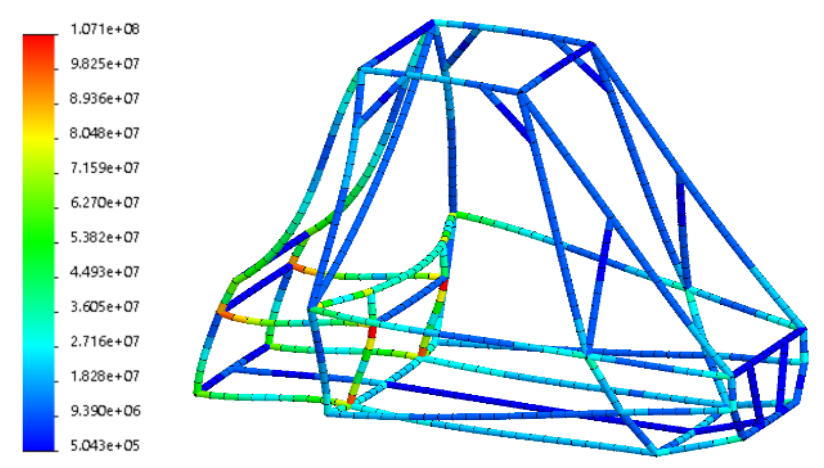

Figura 8 Esfuerzos debido al impacto trasero Fuente: elaboración propia [Solidworks]

\section{Impacto Lateral}

Para calcular la fuerza a aplicar en el impacto lateral se utilizó la masa total del chasis + masa total del motor + masa total de la batería. La suma de todas ellas se multiplicó por una fuerza $\mathrm{G}$ de 2.5 de acuerdo a la fomula de BAJA SAE. $\mathrm{F}=$ ma $140 * 2.5 * 9.1=3500 \mathrm{~N}$ (aprox) aplicamos $3500 \mathrm{~N}$ en la parte lateral izquierda del chasis divididas en 4 fuerzas de $875 \mathrm{~N} \mathrm{c/u}$, se realizaron 4 sujeciones de geometría fija en la parte lateral derecha del chasis. En la figura 9 se muestran las condiciones de carga a las cuales fue sometido el chasis para simular las cargas por impacto lateral.

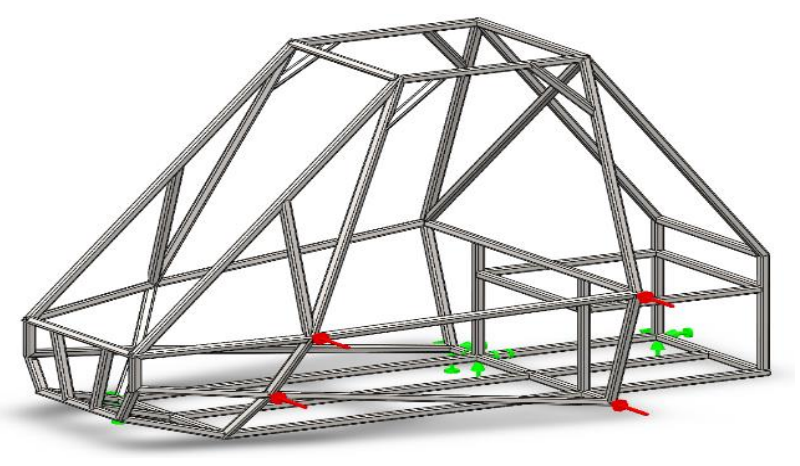

Figura 9 Condiciones de carga debido al impacto lateral Fuente: elaboración propia [Solidworks]

En la figura 10 se observan las deformaciones del chasis bajo las condiciones de carga debido al impacto lateral; también se observó que la máxima deformación fue de $5.46 \mathrm{~mm}$ para el material AISI 1020, 5.32mm para el material AISI 1018 y $5.32 \mathrm{~mm}$ para el material AISI 4130.

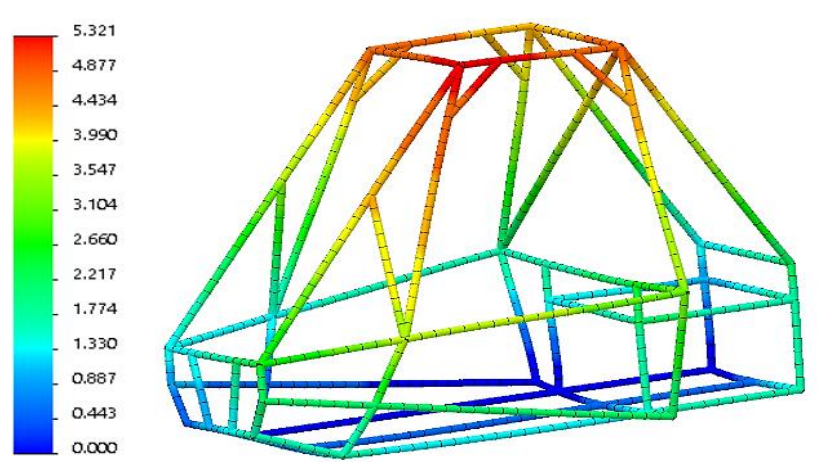

Figura 10 Deformaciones debido al impacto lateral Fuente: elaboración propia [Solidworks]
HUERTA-GAMEZ, Hector, HERRERA-OLIVARES, Edgar Daniel, ZUÑIGA-CERROBLANCO, José Luis y AGUILAR-MORENO, Antonio Alberto. Análisis estructural de chasis prototipo para automóvil tipo SAE BAJA. Revista de Ingeniería Innovativa. 2020 
La figura 11 muestra las zonas donde se encuentra mas esforzado el chasis debido a las cargas por impacto lateral; así mismo se obtuvieron los siguientes resultados: el esfuerzo máximo fue de $184.9 \mathrm{MPa}$ en la estructura quedando a un $52 \%$ en el material AISI 1020, $49 \%$ en el material AISI 1018 y $40 \%$ en el material AISI 4130 de su límite elástico.
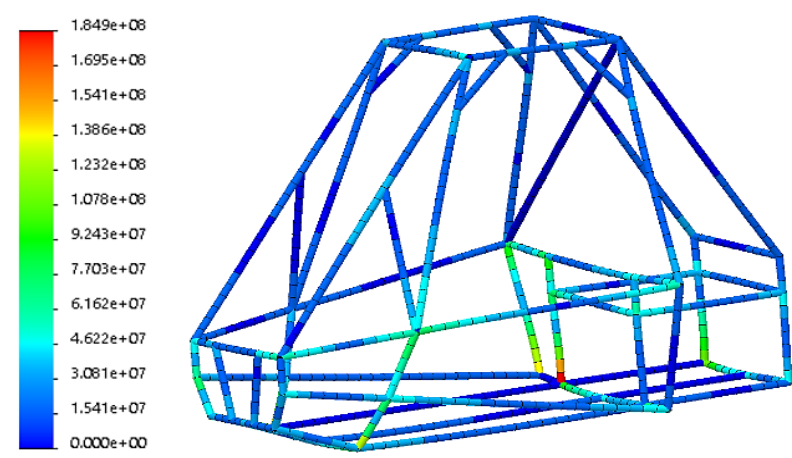

Figura 11 Esfuerzos debido al impacto lateral Fuente: elaboración propia [Solidworks]

\section{Impacto por volcadura}

Para calcular la fuerza a aplicar en el impacto por volcadura se utilizó la masa total del chasis + masa total del motor + masa total de la batería. La suma de todas ellas se multiplicó por una fuerza $\mathrm{G}$ de 4 de acuerdo a la fomula de BAJA SAE. $\mathrm{F}=\mathrm{ma} \quad 140 * 4 * 9.1=5500 \mathrm{~N}$ (aprox) aplicamos $5500 \mathrm{~N}$ en la parte superior del chasis divididas en 2 fuerzas de $2750 \mathrm{~N}$ c/u, se realizaron 8 sujeciones de geometría fija en la parte inferior del chasis. En la figura 12 se muestran las condiciones de carga a las cuales fue sometido el chasis para simular el impacto por volcadura.

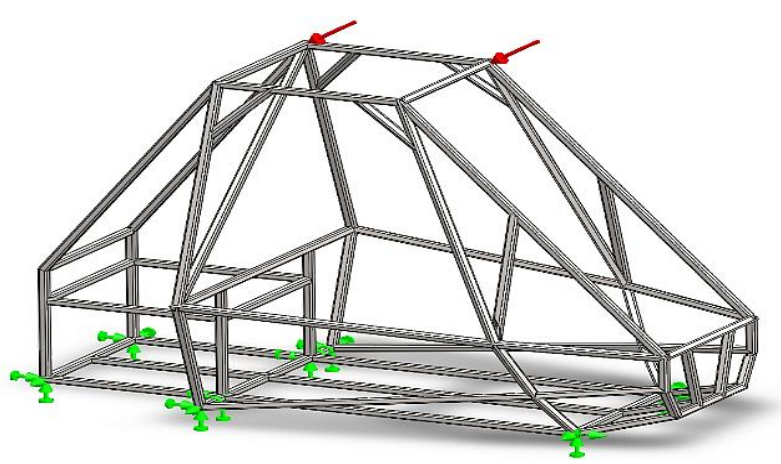

Figura 12 Condiciones de carga debido al impacto por volcadura

Fuente: elaboración propia [Solidworks]
En la figura 13 se observan los desplazamientos del chasis bajo las condiciones de carga debido al impacto por volcadura, las cuales arrojaron los siguientes datos numéricos: la máxima deformación fue de $5.17 \mathrm{~mm}$ para el material AISI 1020, 5.04mm para el material AISI 1018 y $5.04 \mathrm{~mm}$ para el material AISI 4130.

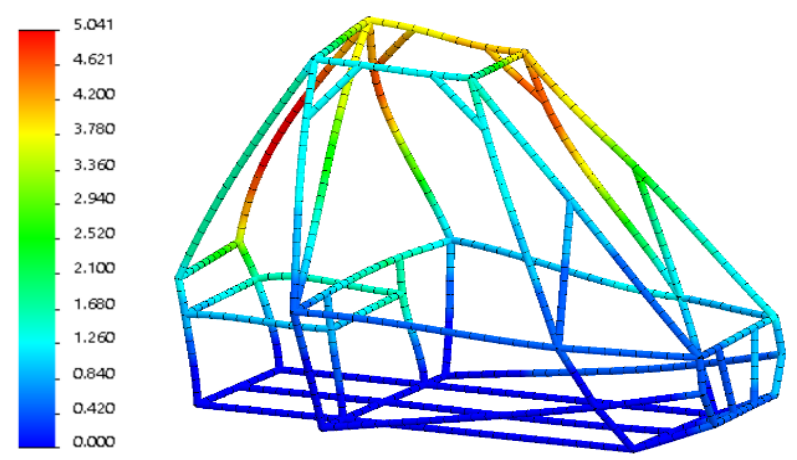

Figura 13 Deformaciones debido al impacto por volcadura

Fuente: elaboración propia [Solidworks]

La figura 14 muestra en qué partes el chasis se encuentra más esforzado debido a las condiciones de carga debido al impacto por volcadura; de igual manera se obtuvieron los siguientes datos numéricos: el esfuerzo máximo fue: $314.4 \mathrm{MPa}$ en la estructura quedando a un $89 \%$ en el material AISI $1020,84 \%$ en el material AISI 1018 y $68 \%$ en el material AISI 4130 de su límite elástico.

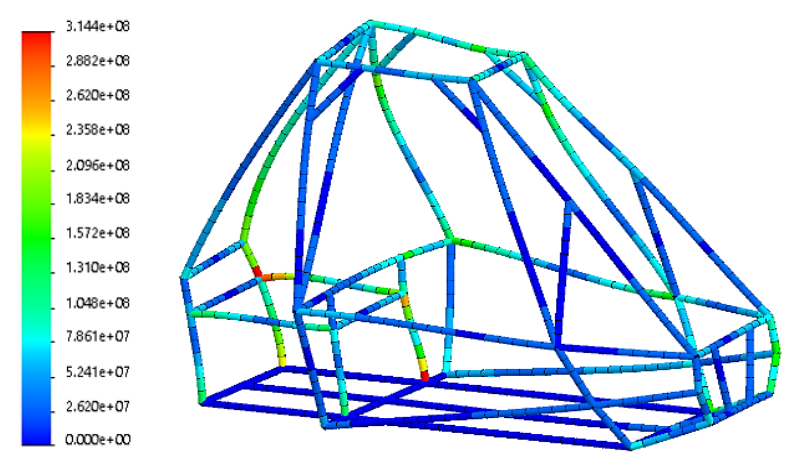

Figura 14 Esfuerzos debido al impacto por volcadura Fuente: elaboración propia [Solidworks]

\section{Impacto por salto delantero}

Para calcular la fuerza a aplicar en el impacto por salto delantero se utilizó la masa total del chasis + masa total del motor + masa total de la batería. 
La suma de todas ellas se multiplicó por una fuerza $G$ de 5 de acuerdo a la fomula de BAJA SAE. F=ma $140 * 5 * 9.1=7000 \mathrm{~N}$ (aprox) aplicamos $7000 \mathrm{~N}$ en la parte donde se situará la suspensión delantera del auto en el chasis divididas en 8 fuerzas de $875 \mathrm{~N} \mathrm{c/u}$, se realizaron 6 sujeciones de geometría fija en la parte trasera inferior del chasis. En la figura 15 se observan las condiciones de carga a las cuales fue sometido el chasis para simular el impacto por salto delantero.

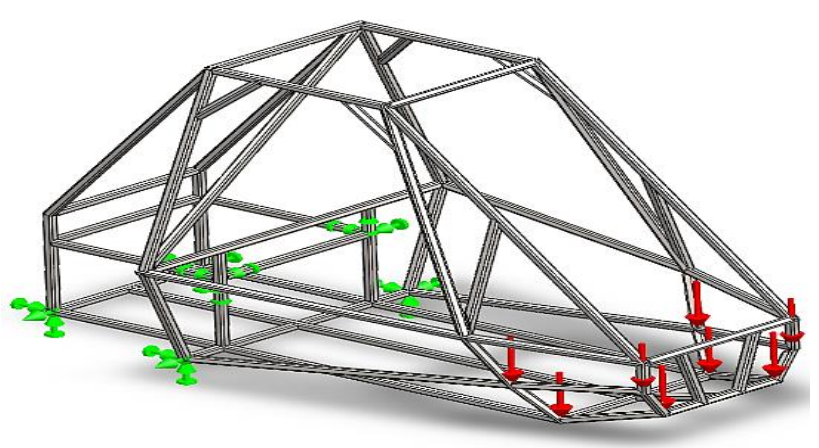

Figura 15 Condiciones de carga debido al impacto por salto delantero

Fuente: elaboración propia [Solidworks]

En la figura 16 se muestra un esquema sobre los desplazamientos del chasis bajo las condiciones de carga debido al impacto por salto delantero, en el cuál se obtuvieron los siguientes datos numéricos: la máxima deformación fue de $8.07 \mathrm{~mm}$ para el material AISI $1020,7.87 \mathrm{~mm}$ para el material AISI 1018 y $7.87 \mathrm{~mm}$ para el material AISI 4130.
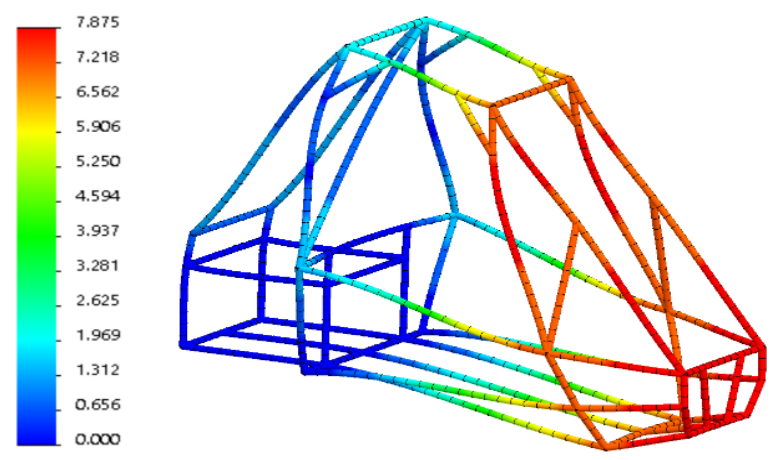

Figura 16 Deformaciones debido al impacto por salto delantero

Fuente: elaboración propia [Solidworks]

La figura 17 muestra las zonas donde el chasis corre mayor riesgo debido al mayor riesgo debido a las condiciones de carga debido al impacto por salto delantero.
Los parámetros encontrados con estas condiciones de carga para el esfuerzo fueron que el esfuerzo máximo fue de $299.5 \mathrm{MPa}$ en la estructura quedando a un $85 \%$ en el material AISI 1020, 80\% en el material AISI 1018 y 65\% en el material AISI 4130 de su límite elástico.
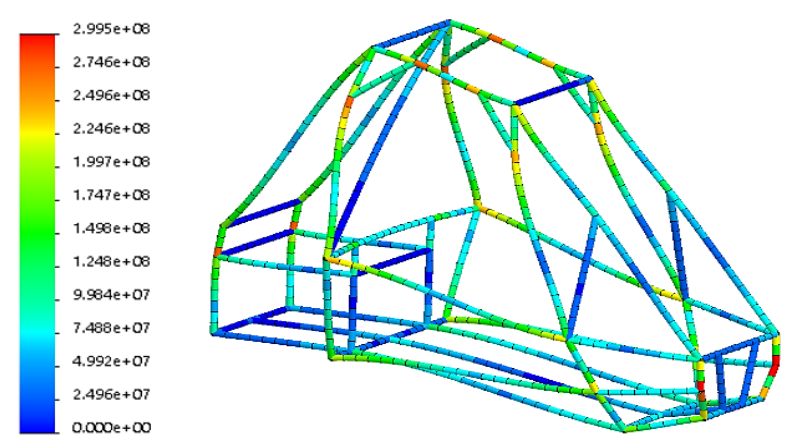

Figura 17 Esfuerzos debido al impacto por salto delantero Fuente: elaboración propia [Solidworks]

\section{Impacto Por Salto Trasero}

Para calcular la fuerza a aplicar en el impacto por salto trasero se utilizó la masa total del chasis + masa total del motor + masa total de la batería. La suma de todas ellas se multiplicó por una fuerza $\mathrm{G}$ de 2.5 de acuerdo a la fomula de BAJA SAE. $\mathrm{F}=\mathrm{ma} 140 * 2.5 * 9.1=3500 \mathrm{~N}$ (aprox) aplicamos $3500 \mathrm{~N}$ en la parte donde se situará la suspensión trasera del auto en el chasis divididas en 4 fuerzas de $875 \mathrm{~N} \mathrm{c/u}$, se realizaron 8 sujeciones de geometría fija en la parte delantera inferior del chasis. En la figura 18 se muestran las condiciones de carga a las cuales fue sometido el chasis para simular el impacto por salto trasero.

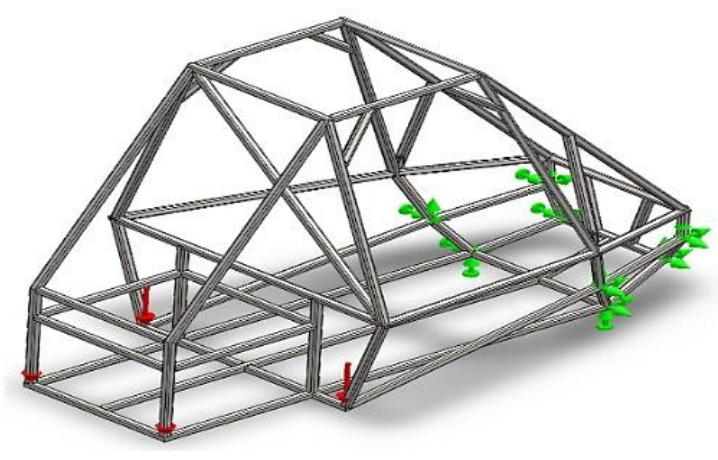

Figura 18 Condiciones de carga debido al impacto por salto trasero

Fuente: elaboración propia [Solidworks] 
En la figura 19 se muestra un esquema sobre los desplazamientos del chasis bajo las condiciones de carga debido al impacto por salto trasero, dentro del cual se obtuvieron los siguientes resultados: la máxima deformación fue de $7.39 \mathrm{~mm}$ para el material AISI 1020 , $7.20 \mathrm{~mm}$ para el material AISI 1018 y $7.20 \mathrm{~mm}$ para el material AISI 4130.
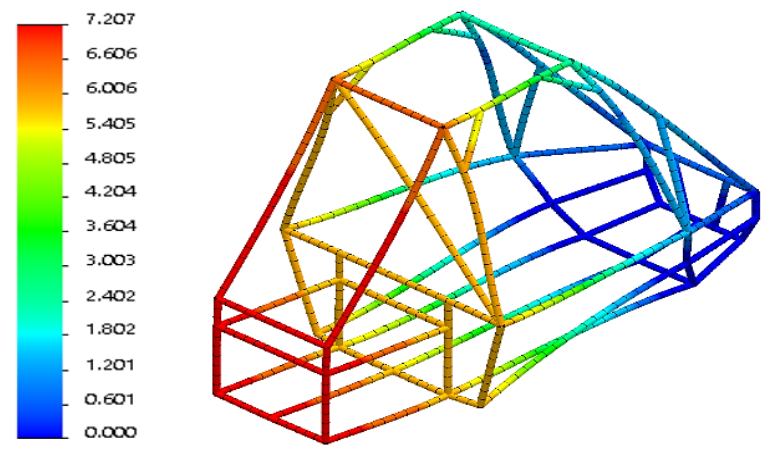

Figura 19 Deformaciones debido al impacto por salto trasero

Fuente: elaboración propia [Solidworks]

La figura 20 muestra en qué secciones el chasis se encuentra más esforzado debido a las condiciones de carga debido al impacto por salto trasero; dentro de dicha simulación se obtuvieron los siguientes parámetros: el esfuerzo máximo fue de $328.7 \mathrm{MPa}$ en la estructura quedando a un $93 \%$ en el material AISI 1020, $88 \%$ en el material AISI 1018 y $71 \%$ en el material AISI 4130 de su límite elástico.
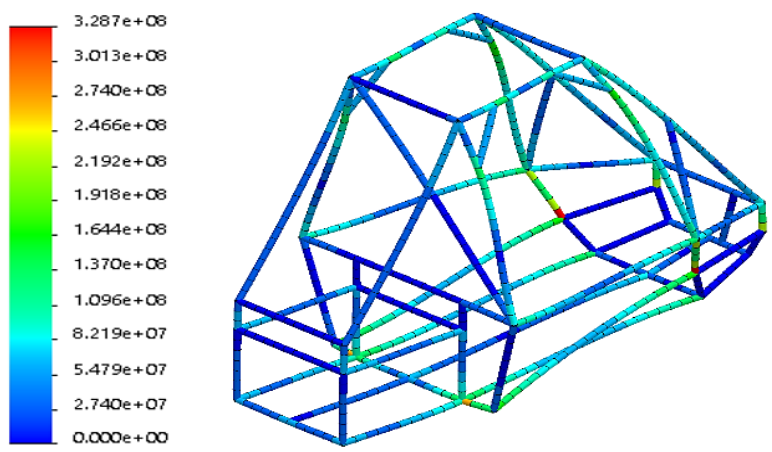

Figura 20 Esfuerzos debido al impacto por salto trasero Fuente: elaboración propia [Solidworks]

\section{Resultados}

En las tablas 3 y 4 se muestran en forma de resumen todos los resultados de las pruebas en cada uno de los materiales, tanto para desplazamientos como para esfuerzos, respectivamente.

\begin{tabular}{|l|r|r|r|}
\hline \multicolumn{1}{|c}{ Análisis } & \multicolumn{1}{c}{$\begin{array}{c}\text { AISI } \\
\mathbf{1 0 2 0}\end{array}$} & \multicolumn{1}{c|}{$\begin{array}{c}\text { 1018 } \\
\text { AISI }\end{array}$} & \multicolumn{1}{c|}{$\mathbf{4 1 3 0}$} \\
\hline Impacto Frontal & 3.68 & 3.59 & 3.59 \\
\hline Impacto Trasero & 1.61 & 1.57 & 1.57 \\
\hline Impacto Lateral & 5.46 & 5.32 & 5.32 \\
\hline $\begin{array}{l}\text { Impacto Por } \\
\text { Volcadura }\end{array}$ & 5.17 & 5.04 & 5.04 \\
\hline $\begin{array}{l}\text { Impacto Por Salto } \\
\text { Delantero }\end{array}$ & 8.07 & 7.87 & 7.87 \\
\hline $\begin{array}{l}\text { Impacto Por Salto } \\
\text { Trasero }\end{array}$ & 7.39 & 7.20 & 7.20 \\
\hline
\end{tabular}

Tabla 3 Resultados de desplazamientos en mm Fuente: elaboración propia [Word]

\begin{tabular}{|l|r|r|r|r|}
\hline \multicolumn{1}{|c|}{ Análisis } & \multicolumn{1}{c}{$\begin{array}{c}\text { AISI } \\
\mathbf{1 0 2 0}\end{array}$} & \multicolumn{1}{c}{ AISI } & \multicolumn{1}{c|}{$\begin{array}{c}\text { AISI } \\
\mathbf{1 0 1 8}\end{array}$} & $\begin{array}{c}\text { Esfuerzo } \\
\text { (MPa) }\end{array}$ \\
\hline $\begin{array}{l}\text { Impacto } \\
\text { Frontal }\end{array}$ & $29 \%$ & $27 \%$ & $22 \%$ & 102.4 \\
\hline $\begin{array}{l}\text { Impacto } \\
\text { Trasero }\end{array}$ & $30 \%$ & $28 \%$ & $23 \%$ & 107.1 \\
\hline $\begin{array}{l}\text { Impacto } \\
\text { Lateral }\end{array}$ & $52 \%$ & $49 \%$ & $40 \%$ & 184.9 \\
\hline $\begin{array}{l}\text { Impacto } \\
\text { Por } \\
\text { Volcadura }\end{array}$ & $89 \%$ & $84 \%$ & $68 \%$ & 314.4 \\
\hline $\begin{array}{l}\text { Impacto } \\
\text { Por Salto } \\
\text { Delantero }\end{array}$ & $85 \%$ & $80 \%$ & $65 \%$ & 299.5 \\
\hline $\begin{array}{l}\text { Impacto } \\
\text { Por Salto } \\
\text { Trasero }\end{array}$ & $93 \%$ & $88 \%$ & $71 \%$ & 328.7 \\
\hline
\end{tabular}

Tabla 4 Resultados de esfuerzos en \% de acuerdo con el límite elástico de cada material

Fuente: elaboración propia [Word]

\section{Conclusiones}

Con los resultados obtenidos en los diferentes análisis se procede con la fabricación del chasis con el acero AISI 1018 debido a su buen comportamiento en las pruebas, en gran similitud a la AISI 4130, con un costo reducido.

Se continuará trabajando en el miniauto para cualquier ajuste que se realice al chasis, mejorando de esta forma el diseño, además de continuar trabajando en un mediano plazo con el sistema de dirección del vehiculo.

\section{Referencias}

[1] Nawani, S., Bisht, K., \& Chopra, S. . (2014). FEM Analysis of BAJA Chassis. Octubre, 2019, de International Journal of Aerospace and Mechanical Engineering Sitio web: http://ijamejournals.com/pdf/FEM\%20Ana lysis\%20of\%20BAJA\%20Chassis.pdf

HUERTA-GAMEZ, Hector, HERRERA-OLIVARES, Edgar Daniel, ZUÑIGA-CERROBLANCO, José Luis y AGUILAR-MORENO, Antonio Alberto. Análisis estructural de chasis prototipo para automóvil tipo SAE BAJA. Revista de Ingeniería Innovativa. 2020 
[2] Shivam, M.. (2017). Static Analysis of the Roll Cage of an All-Terrain Vehicle (SAE BAJA). Octubre, 2019, de International Research Journal of Engineering and Technology (IRJET) Sitio web: https://www.irjet.net/archives/V4/i9/IRJET -V4I9157.pdf

[3] Hemant, K., Nagendra, P., \& Deepty, V.. (2011). Finite Element Analysis of Impact on Chassis \& Frame of a Mini Baja Vehicle. Octubre, 2019, de International Journal of Emerging trends in Engineering and Development Sitio web: https://pdfs.semanticscholar.org/e9dd/04f94 4cf8f20624eb9c3d8010c38c490d386.pdf?_ $\mathrm{ga}=2.240623043 .17508895 .1593288825$ 1473742366.1593288825

[4] Krishna, J., Ambesh, S., \& Prabhudev, M.. (2017). Design and Analysis of Chassis for SAE BAJA Vehicle. Octubre, 2019, de IOSR Journal of Engineering (IOSR JEN) Sitio web: https://www.iosrjen.org/Papers/Conf.19017 -2019/Volume-4/7.\%2051-57.pdf

[5] BAJA SAE. (2019). Collegiate Design Series Baja SAE® Rules 2020. Octubre, 2019, de BAJA SAE Sitio web: https://www.google.com/url?sa=t\&rct=j\&q= \&esrc $=$ s\&source $=$ web $\& c d=\& v e d=2$ ahUKE wiJgP_66LqAhUHCM0KHUzxCpwQFjA AegQIARAB\&url=https\%3A\%2F\%2Fwww .bajasae.net\%2Fcdsweb\%2Fgen\%2FDownlo adDocument.aspx\%3FDocumentID\%3Dcd6 d65ab-b69b-4e1e-990d-

f961b9f4cccb\&usg=AOvVaw0_KbKi-

6AisT0HQ_82ZxE6
HUERTA-GAMEZ, Hector, HERRERA-OLIVARES, Edgar Daniel, ZUÑIGA-CERROBLANCO, José Luis y AGUILAR-MORENO, Antonio Alberto. Análisis estructural de chasis prototipo para automóvil tipo SAE BAJA. Revista de Ingeniería Innovativa. 2020 